\title{
A BLUEPRINT FOR TELEREHABILITATION GUIDELINES
}

\author{
DAVID BRENNAN, MBE¹, LYN TINDAll, PHD², DEBORAH THEODOROS, PHD³, \\ JANET BROWN, MA*4, MICHAEL CAMPBELL, MS5, DIANA CHRISTIANA, MAT ${ }^{6}$, \\ DAVID SMITH ${ }^{7}$, JANA CASON, DHS ${ }^{8}$ ALAN LEE, DPT ${ }^{9}$
}

\author{
${ }^{1}$ NATIONAl ReHABILITATION HOSPITAl, WASHINGTON, DC, 2 DePARTMENT OF VETERANS AFFAiRS \\ MEDICAL CENTER, LEXINGTON, KY, ${ }^{3}$ THE UNIVERSITY OF QUEENSLAND, QUEENSLAND, AUSTRALIA, \\ ${ }^{4}$ AMERICAN SPEECH-LANGUAGE-HEARING ASSOCIATION, ROCKVILLE, MD, ${ }^{5}$ UNIVERSITY OF NORTH \\ CAROLINA AT GREENSBORO, BROWNS SUMMIT, NC, ${ }^{6}$ CLINICAL COMMUNICATIONS, SUGAR LAND, TX, \\ 7 Telehealth Resource Center - MarquetTe General Hospital, MarguetTe, Mi, ${ }^{8}$ AuerbaCH \\ SCHOOL OF OCCUPATIONAL THERAPY, SPALDING UNIVERSITY, LOUISVILLE, KY, 9 MOUNT ST. MARY'S \\ COllege, LOS ANGeles, CA
}

\section{ABSTRACT}

Telerehabilitation refers to the delivery of rehabilitation services via information and communication technologies. Clinically, this term encompasses a range of rehabilitation and habilitation services that include assessment, monitoring, prevention, intervention, supervision, education, consultation, and counseling. Telerehabilitation has the capacity to provide service across the lifespan and across a continuum of care. Just as the services and providers of telerehabilitation are broad, so are the points of service, which may include health care settings, clinics, homes, schools, or community-based worksites. This document was developed collaboratively by members of the Telerehabilitation SIG of the American Telemedicine Association, with input and guidance from other practitioners in the field, strategic stakeholders, and ATA staff. Its purpose is to inform and assist practitioners in providing effective and safe services that are based on client needs, current empirical evidence, and available technologies. Telerehabilitation professionals, in conjunction with professional associations and other organizations are encouraged to use this document as a template for developing discipline-specific standards, guidelines, and practice requirements.

Keywords: Telerehabilitation, telepractice, rehabilitation, American Telemedicine Association

\section{INTRODUCTION}

The Telerehabilitation Special Interest Group of the American Telemedicine Association is comprised of practitioners in health and education, and technology specialists who are engaged in applying telecommunications and health information technologies to improve access to rehabilitation and educational services, and to support independent living. This document was developed collaboratively by members of the Telerehabilitation SIG, with input and guidance from other practitioners in the field, strategic stakeholders, and ATA staff.

The purpose of this guide is to inform and assist practitioners in providing effective and safe services that are based on client needs, current empirical evidence, and available technologies. The material in this guide addresses general principles and not specific practice guidelines for telerehabilitation and is not intended to replace the primary practitioner's clinical, educational, or technology decision-making about the appropriate course of action/management of any client. Inherent within this document is the recognition that safe and effective telerehabilitation practice requires specific training, skills and techniques as documented below. Furthermore, the material in this guide should not be interpreted, nor used, as a legal standard of care.

\section{SCOPE AND DEFINITIONS}

Telerehabilitation refers to the delivery of rehabilitation services via information and communication technologies. Clinically, this term encompasses a range of rehabilitation and habilitation services that include assessment, monitoring, prevention, intervention, supervision, education, consultation, and counseling. Telerehabilitation services are delivered to adults and children by a broad 
range of professionals that may include, but is not limited to, physical therapists, speech-language pathologists, occupational therapists, audiologists, rehabilitation physicians and nurses, rehabilitation engineers, assistive technologists, teachers, psychologists, and dieticians. As other personnel such as paraprofessionals, family members, and caregivers may assist during telerehabilitation sessions, for the purposes of this document, the term 'professionals' will be used to denote professional providers of telerehabilitation services. The term 'clients' will be used to refer to all recipients of telerehabilitation services and is intended to include both patients in medical settings, and children and adults who receive services outside the medical sphere, for example, in schools or at home.

Telerehabilitation has the capacity to provide service across the lifespan and across a continuum of care. Just as the services and providers of telerehabilitation are broad, so are the points of service, which may include health care settings, clinics, homes, schools, or community-based worksites. Terminology used to describe telerehabilitation is similarly broad. Some terms are used specifically to refer to individual rehabilitation disciplines, such as telespeech (speech-language pathology) or teleOT (occupational therapy). More generic terms, such as teletherapy and telepractice are also used, allowing for a broader focus on populations and activities, such as educational settings and wellness promotion in addition to rehabilitation. It is not the intent of this document to resolve the debate over terminology; rather the goal is to provide consistency across applications, regardless of the vocabulary used. For the purposes of this document, the term 'telerehabilitation' will be used, and the reader is reminded that terminology may differ according to the application and location.

\section{KEY PRINCIPLES}

The following information represents key administrative, clinical, technical, and ethical principles that should be considered in the course of providing telerehabilitation services. They are based primarily on the American Telemedicine Association's Core Standards for Telemedicine Operations, and describe additional considerations that are present across applications within telerehabilitation and its related fields. As education and advocacy are central to the continued growth of telerehabilitation, this document can and should be used as a tool to educate members of the medical and education professions, students, stakeholders, administrators, legislators, and community members. Telerehabilitation professionals, in conjunction with professional associations and other organizations, are encouraged to use this document as a template for developing discipline-specific standards, guidelines, and practice requirements. A bibliography of research in the field can be found on the webpage for the Telerehabilitation Special Interest Group of the American Telemedicine Association (www.americantelemed.org).

\section{ADMINISTRATIVE PRINCIPLES:}

- Organizations and/or professionals shall comply with national, state, local and other credentialing, privileging, and regulatory requirements for licensure, certification, and for the use of telerehabilitation.

- Organizations and/or professionals shall be aware of their locus of accountability and any requirements (including those for liability insurance) that apply when practicing telerehabilitation. This includes credentialing requirements at the site where the practitioner is located and the site where the client is located (which may be different states or jurisdictions), in compliance with regulatory and accrediting agencies.

- If required, organizations and/or professionals shall use billing and coding processes that designate that telerehabilitation services have been provided (e.g. through the use of a modifier) as required per guidelines of the payer.

- Organizations and/or professionals shall have traceable documentation that a telerehabilitation session occurred with a client. This documentation should be available to both the referring and consulting sites as appropriate.

- Organizations and/or professionals shall be aware of the advanced requirements for privacy and confidentiality associated with provision of services through telehealth technology at both the originating site and remote setting.

- Organizations and/or professionals shall determine requirements for documentation, storage, and retrieval of client records to protect the client's personal health information in accordance with federal and state regulations (e.g. HIPAA, FERPA, etc.). Specific guidelines shall be in place to address access to client records so as to ensure that unauthorized users cannot access, alter, tamper with, destroy or otherwise misuse client information.

- Organizations and/or professionals shall have a mechanism in place for ensuring that clients are aware of their rights and responsibilities with respect to accessing health care via telehealth technologies, including the process for communicating complaints.

- Organizations and/or professionals shall ensure that an appropriate facilitator is available when necessary to meet client and provider needs before, during, and after the telerehabilitation encounter.

- Organizations and/or professionals engaged in telerehabilitation research shall ensure the protection of participants in research protocols. Research protocols shall be approved by local Institutional Review Board(s) and be in compliance with relevant legislation, regulations, and other requirements for supporting participant decision-making and informed consent as well as safeguarding of Protected Health Information.

- Organizations and/or professionals shall ensure they have appropriate technology expertise during planning and start-up phases of a telerehabilitation program. Reasonable care and diligence shall be used when 
selecting equipment for evaluating or treating a client. Practitioners need to take appropriate measures to familiarize themselves with equipment and safety issues with client use. To help ensure success, it is essential that all telerehabilitation providers use appropriate planning prior to delivering services and are fully aware of the capabilities and limitations of the equipment they intend to use, and the impact it may have on service delivery.

- Organizations and/or professionals shall have in place a systematic quality improvement and performance management process that complies with any organizational, regulatory, or accrediting, requirements for outcomes management.

- Organizations and/or professionals that engage in collaborative partnerships shall be aware of applicable legal and regulatory requirements for appropriate written agreements, memorandum of understanding, or contracts. Those contracts, agreements, etc., shall be based on the scope and application of the telerehabilitation services offered and shall address the administrative, clinical, technical, and ethical requirements outlined in this document, as relevant, for all parties named.

\section{CLINICAL PRINCIPLES:}

- Professionals shall be guided by existing discipline and national clinical practice guidelines when practicing via telehealth. Where guidelines, position statements, or standards for telerehabilitation exist from a professional organization or society (e.g. American SpeechLanguage Hearing Association (ASHA), American Physical Therapy Association (APTA), and American Occupational Therapy Association (AOTA)), these shall be reviewed and appropriately incorporated into practice. Given the variability of rehabilitation clients, candidacy and appropriateness for telerehabilitation should be determined on a case-by-case basis with selections firmly based on clinical judgment, client's informed choice, and professional standards of care.

- As in all settings, professionals shall have the appropriate education, training/orientation, and ongoing continuing education/professional development to insure they possess the necessary competencies for the safe provision of quality health services. Providers of telerehabilitation should have competence in the use of equipment, as well as considerations related to clients with cognitive, physical, or perceptual impairments.

- Delivery of services via telerehabilitation, whether interactive or store-and-forward, may require modifications to treatment material, techniques, equipment, and setting. Regardless of any modifications made, professionals shall deliver services in accordance with professional standards of care and the principles of evidence-based practice (i.e., current best evidence, clinical expertise, and client values and goals). In telerehabilitation encounters, these modifications may include: using additional support staff at the remote site to assist the client with a physical activity or perform a hands-on assessment, digitally reproducing treatment materials for delivery via data sharing or presentation tools, or temporary modification of client environment.

- Professionals shall ensure that all persons in the therapy room at both sites are identified to all participants prior to initiation of the telerehabilitation session. Disclosure of individuals attending the session may be accomplished by announcing their presence.

\section{TECHNICAL PRINCIPLES:}

- Organizations and/or professionals shall ensure that equipment sufficient to support diagnostic and/or treatment needs is available and functioning properly at the time of clinical encounters. Beyond basic telehealth technology, additional equipment is often required to provide telerehabilitation services. This equipment will vary based on application and may include, for example, a sound level meter to measure intensity of speech, an audiometer for hearing assessment, or online measurement tools and sensors to measure force or position during a physical therapy assessment.

- Organizations and/or professionals shall comply with all relevant laws, regulations, and codes for technology and technical safety.

- Organizations and/or professionals shall comply with federal and state regulations for protection of client health information and to ensure the physical security of telehealth equipment and the electronic security of data storage, retrieval, and transmission. Methods for protection of health information include the use of authentication and/or encryption technology, and limiting access to need-to-know (availability for those people who do require access).

- Organizations and/or professionals shall ensure that all personnel who use telehealth equipment to deliver information or services are trained in equipment operation and troubleshooting. Procedures shall also be in place to ensure the safety and effectiveness of equipment through on-going maintenance. Providers of telerehabilitation must know how to operate videoconferencing peripherals, such as document cameras and data sharing tools, to incorporate other treatment materials into a session and configure audio/video signals so they are appropriate for clients with visual or hearing impairments.

- Organizations and/or professionals shall have strategies in place to address the environmental elements of care, including the physical accessibility of the treatment space as well as usability of equipment. This is essential in telerehabilitation applications as considerations must be made for clients who have a variety of impairments in areas such as fine/gross motor skill, cognition, speech, language, vision, or hearing.

- Organizations and/or professionals shall have infection control policies and procedures in place for the use of telehealth equipment and client peripherals that comply with organizational, legal, and regulatory requirements. In particular, mechanisms shall be in place for the cleaning/ sterilization of equipment for re-use by multiple clients. 


\section{ETHICAL PRINCIPLES:}

- The use of telehealth technology to deliver rehabilitation services requires consideration of professional ethical principles. Organizations and professionals that adhere to ethical principles of telerehabilitation shall:

- Incorporate organizational values and ethics into policy and procedures documents for telerehabilitation

- Comply with professional codes of ethics

- Inform clients of their rights and responsibilities when receiving rehabilitation through telehealth technology, including their right to refuse

- Have in place a formal process for resolving ethical issues as well as policies that identify, eliminate, and reduce conflicts of interests associated with the provision of telerehabilitation services

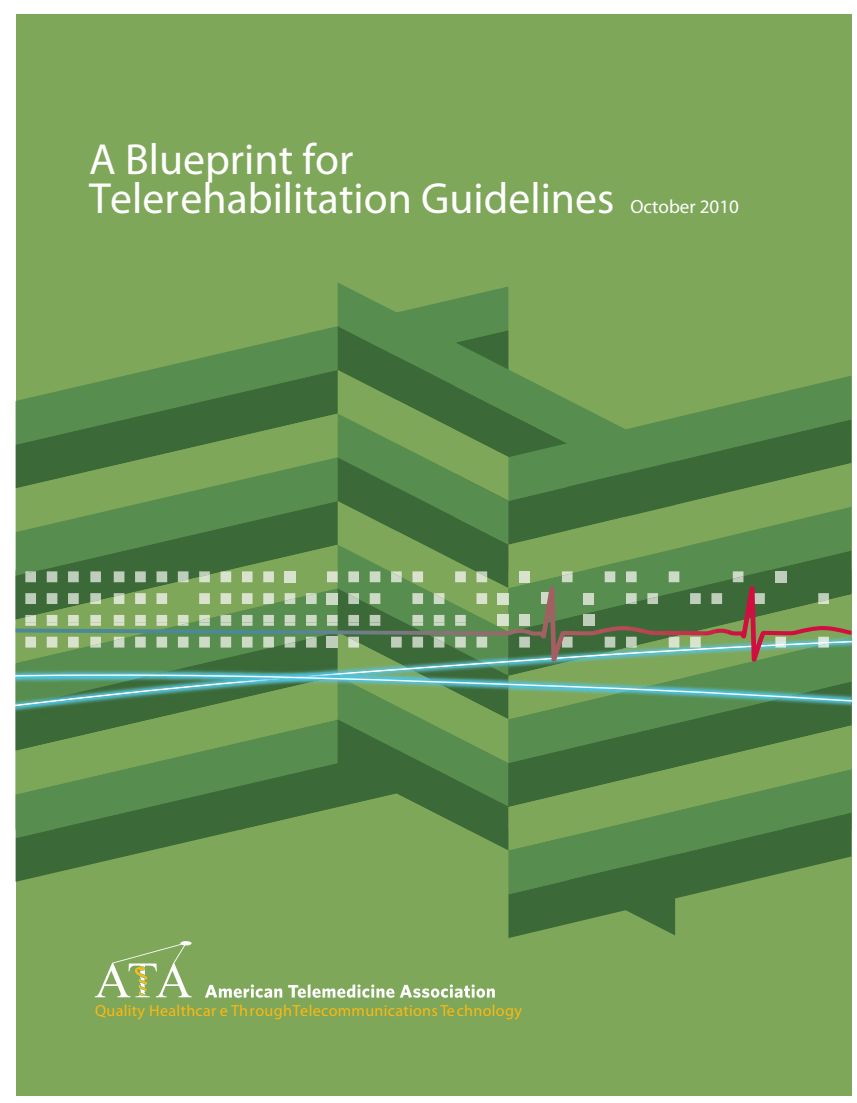

\section{ACKNOWLEDGEMENTS}

This document was developed by a committee chaired by David Brennan, MBE and Lyn Tindall, PhD as part of the Telerehabilitation Special Interest Group of the American Telemedicine Association. The authors gratefully acknowledge the contributions of the following Working Group Members [WG], Consultants [C],

Reviewers [R], Telerehabilitation Special Interest Group
Chair [TR], ATA Standards and Guidelines Committee Member [SG], and ATA Staff [S]; Nina Antoniotti, RN, MBA, PhD, [Chair, SG], Director of Telehealth, Marshfield Clinic, Marshfield, Wl; Jordana Bernard, MBA, [S], Senior Director, Program Services, American Telemedicine Association, Washington DC; Anne Burdick, $\mathrm{MD}, \mathrm{MPH}$, [SG], Associate Dean for Telemedicine and Clinical Outreach, Professor of Dermatology, Director, Leprosy Program, University of Miami Miller School of Medicine, Miami, FL; Jerry Cavallerano, PhD, OD, [SG], Staff Optometrist, Assistant to the Director, Joslin Diabetes Center, Beetham Eye Institute, Boston, MA; Ellen Cohn, PhD, CCC-SLP, [Chair, TR, WG], Associate Dean for Instructional Development, School of Health and Rehabilitation Sciences, University of Pittsburgh, Pittsburgh, PA; Paul Cox, MSEE, [WG], President, PERL Research, Huntsville, AL; Mary Fran Delaune, PT, MPT, [R], Director, Practice Department, American Physical Therapy Association, Alexandria, VA; Matt Elrod, PT, DPT, MEd, NSC, [R], Associate Director, Practice Department, American Physical Therapy Association, Alexandria, VA; Brian Grady, MD, [SG], VISN5 TMH Lead \& Director, TeleMental Health, School of Medicine, University of Maryland, Baltimore, MD; Elizabeth Krupinski, PhD, [Vice Chair, SG], Associate Director, Program Evaluation, University of Arizona, Arizona Telemedicine Program, Department of Radiology, Research Professor, Department of Radiology Research, Tucson, AZ; Jonathan D. Linkous, MPA, [S], Chief Executive Director, American Telemedicine Association, Washington, DC; Michael Pramuka, PhD, CRC, [WG], Rehabilitation Counselor, Walter Reed Medical Center, Washington, DC; Richard Schein, PhD, [WG], Postdoctoral Associate, School of Health and Rehabilitation Sciences, University of Pittsburgh, Pittsburgh, PA; Lou Theurer, [SG], Grant Administrator, Burn Telemedicine Program, University of Utah Health Sciences Center, Salt Lake City, UT; and Jill Winters, PhD, RN, [WG, SG], Dean and Professor, Columbia College of Nursing, Milwaukee, WI. 